\section{Homo Videns}

\section{Gabor B. Levy}

After the trauma of birth, a baby is suddenly in a strange new world. The first order of the day is to make sense out of the jumble of signals in order to form an image of what is out there. This image building proceeds with increased accuracy throughout adolescence. For those who labor in science the process never stops. They continue to try to refine their mental image of nature throughout their professional lives. In this effort, vision is the most valuable natural sense. In a happy blend of poetry and science, Diane Ackerman in her book, A Natural History of Senses, calls eyes "the great monopolist of our senses." It is, in fact, hard to imagine experimental science without the power of vision. By the same token, any enhancement of visual acuity will promote the precision of our image of nature - the very essence of science.

It is a logical sequel then, that the invention of the telescope and microscope during the first years of the 17th century coincided with the rapid growth of modern natural sciences. The telescope, in particular, served to refine our view of the universe of which we are such a very small part. This was hard to accept because it ran counter to the established idea that our earth and ourselves are the center of creation. In fact, to this very day many people cannot cope with the idea. But with the aid of telescopes we can actually see the truth.

The microscope had an equally dramatic impact by making it possible for one to see a completely unexpected abundance of teeming life, invisible to the naked eye. The fine structure of familiar objects also became visible. This visibility explained many of the properties of the objects. The advantages were so obvious that microscopy developed steadily and had an impact on all branches of science. Microscopy also gave rise to entirely new disciplines such as bacteriology. By the end of the last century, affordable microscopes became available that performed to the theoretical limit of refracting optics: a resolution of approximately $0.2 \mu \mathrm{m}$. This amounts to an enormous increase in visual acuity of over a thousand-fold. There the matter rested until about 50 years ago.

Then a new principle arose, that of electron optics. It was based on the insight of de Broglie, who postulated that an electron beam would have wave properties similar to electromagnetic waves such as light. Because of the very short virtual wavelength, electron microscopes were not subject to the limit of light optics. Even the early commercial instruments had up to a hundred times better resolution. Working with them was as exciting as it must have been with the 17 th century microscopes, because new invisible organisms such as viruses and bacteriophages, whose existence had previously been only conjectured, could now be seen. Within the same time frame, electronics developed apace, and it became possible to synthesize images by point-wise scanning. Although the newly developed scanning reflection electron microscopes did not enhance resolution, they produced an unprecedented three-dimensional view on a molecular scale. The results were not only of psychological and esthetic advantage, but of scientific value as well.

The next big leap forward came unanticipated, with the invention of scanning tunneling microscopy just a few years ago. It is not an optical method, but it results in a visual display of atomic dimensions of the scanned sample surface. It operates on a sort of tactile principle, although with the large array of scanning probes that were developed, it is hard to categorize. Probes using almost any physical principle can be made: atomic force, conductivity, nuclear magnetic resonance, friction, temperature, and recently even chemical forces. The gain in resolution was considerable and critical, because by reaching down to 10 picometers, we moved from molecular to atomic levels. The impact of atomic force microscopy was particularly felt in biological research, because in contrast to electron microscopy, it became possible to explore materials that were not denatured. One of the most exciting new reports was the observation of a lysozyme molecule distending as it ingested a substrate. It was reminiscent of the image of a snake swallowing a mouse.

The development of these new technologies made possible by electronic and computational abilities reflected back onto optical microscopy, with new technologies and some new principles. The spectral ranges were extended from X-ray to infrared and Raman imaging. Fluorescence microscopy is in widespread use and now includes simultaneous multiwavelength analysis. One of the defects of light microscopy is the shallow depth of focus. This causes the superposition of blurred images onto the field in focus. Confocal scanning microscopy has remedied this defect and facilitates three-dimensional scans. A surprising novel technique is an optical paradox, since it breaks through the limit of resolution by bypassing diffraction optics. The technique is near-field microscopy, which already offers resolutions 20 times better than that of conventional light microscopes.

Apace with the development of direct imaging, computer technology also impacted chemical and structural analysis. Electron and X-ray diffraction methods improved enormously. The exact configurations in biological macromolecules became easily accessible, which had a great impact on chemical and pharmaceutical research. Computer-aided chemistry is becoming as commonplace as computer-aided design/manufacturing (CAD/CAM). The latter truly revolutionized the drafting rooms, where today cathode ray displays are in place of drafting boards. In the same way, chemists now can view organic molecules, inspection them and turning them around by computer keystrokes. Then they can devise the proper fit for new molecules.

This new type of visualization extends to the human scale in medicine. The venerable $X$-ray imaging of the beginning of this century has now been extended to three-dimensional tomography. To this has been added positron and nuclear magnetic imaging, which allows the visualization of soft tissues, and even of biological processes in vivo. Magnetic resonance imaging also has the advantage of being nonionizing. Another new nonionizing method is sonography, in which the image is generated essentially by differences in elasticity. Today the physician can actually see inside the patient, not only by images generated by these scanning methods, but also directly, by inserting fiber optic devices through tiny incisions. Laparoscopy permits invasive abdominal operations with a minimum of trauma. Even after a complete gall bladder removal, the patient ends up with a few tiny plasters and is sent home.

Turning from the human to the global scale, satellites made it possible to view our Earth from space. It must have been shattering to see the Earth from outer space as an illuminated globe floating in the dark. Even the photographs were thrilling. The sober scientific benefits were equally stunning. The recent geographic and ecological surveys would have been impossible without the space program. A new technology is in place, called global positioning system (GPS), by which we can locate ourselves within meters by using signals from 24 orbiting satellites. Today we can actually see ourselves anywhere on sea or land on projected nautical or land maps, and chart our course along waterways, highways, or city streets.

We can now turn to inspecting the vast universe by telescopes. Instruments with both refracting and reflecting optics were used early on, but reflectors won out because they are free of chromatic aberrations. Because it is the size of the primary mirror that limits resolution, technology concentrated on the production of giant telescopes. Finally, 200-in. telescopes such as the one at Mt. Palomar represented a practical limit. This limit was recently breeched by the novel design of the Keck telescope, which is a composite of 36 hexagonal mirror segments yielding a telescope of virtually 400 in. or $10 \mathrm{~m}$ in diameter. Astronomy recently also expanded to radio frequencies. There, too, larger and larger diameters were sought. However, because of the new capabilities of exquisite synchronization, it has become commonplace to have several smaller dish antennas operate as one very large antenna. The newest very long baseline array (VLBA) of 10 radio antennas are positioned from Hawaii to the Virgin Islands; a virtual radio telescope 8000 miles across. There are further refinements in light microscopy in store. To overcome the cost and complexity of grinding high-precision parabolic mirrors, liquid mirrors of spinning mercury have been proposed. More practical is the proposal to use spherical mirror segments, which are much simpler to produce than parabolic ones, and add small correcting mirrors. A major problem of all telescopes is atmospheric distortion, which is being substantially overcome by active and adaptive optics. The former correct low-frequency wavefront errors (less that $0.01 \mathrm{~Hz}$ ) by flexing the large primary mirror or adjusting the individual segments in the Keck telescope. Adaptive optics correct the rapid (up to $1000 \mathrm{~Hz}$ ) distortions by small 
deformable mirrors in the optical train, using a fixed star as a reference. A totally effective but expensive solution is telescopes operating in space outside the atmosphere, such as the Hubble telescope. Even though it is far from record size, it is capable of gathering clear views from prodigious distances. We are not looking at galaxies some 10 billion light years from us - incredible $10^{25} \mathrm{~m}$ (so large a distance that to describe it, we have to resort to the newest SI prefix of "Yotta", i.e. $10 \mathrm{Ym}$ ). Considering that what we see reflects a view 10 billion years old, the dimensions of these developments can be overwhelming.

This progress started a few hundred years ago, but accelerated during the last century, and increasingly so during the last few years. We had to acquire a dictionary of new words, acronyms, and abbreviations: lasers, fiber optic devices. TEM and SEM, STM and AFM, confocal and near-field microscopy, NMR and MRI, sonograms, GPS, and VLBA. We touched on all of them briefly, but discussed only the highlights. Our visual capabilities have expanded dramatically - spectrally, from the measly $1: 2$ ratio by eye, to some 12 orders of magnitude from gama rays to radio waves. We had a staggering million-fold gain in resolution by microscopy, from $0.1 \mathrm{~mm}$ to $0.1 \mathrm{~nm}$. In far vision, from an angular resolution of about $1^{\circ}$ to better than $0.1^{11}$ with large telescopes - an enhancement of 50,000 , and a stunning enhancement of 100 million with the very long baseline radio interferometry. Of course, our world view changed and sharpened, and our lifestyle changed as well. Today, our restless eyes are riveted to cathode ray displays in our working and leisure hours; equal to the time they are closed during sleep. These are big changes, but human evolution is measured in many millennia, not years. Our species is fixed. But with this vast improvement of our vision, we have transformed ourselves into a virtual new species: from Homo sapiens to Homo videns.

Reprinted from American Laboratory

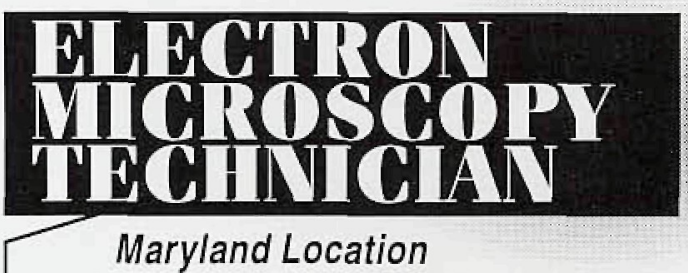

W.L. Gore \& Associates, Inc., is recognized worldwide as a progressive, high-technology manufacturing company. We have an exciting opportunity for an Electron Microscopy Technician at one of our Maryland facilities. Responsibilities include routine production testing via sample prep and microscopy, supporting routine process and product trouble-shooting, operating and maintaining equipment. This opportunity will also involve writing technical reports and other administrative support as necessary.

Requirements include:

- 3-5 years experience in operating and maintaining scanning electron microscopes

Experience with polymeric materials

- Demonstrated experience leading projects and people

- Computer skills

- Willingness to learn other analytical tools

- Excellent interpersonal skills

Qualified applicants, please send or fax your resume to:

W.L. Gore \& Associates, Inc.

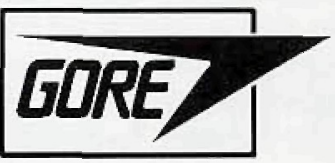

An Equal Opportunity Employer
Attn: 2012ECl

P.O. Box 9206

Newark, DE

19714-9206

Fax: (302) 292-4156

\section{ROBINSON DETECTOR}

\section{WITH ANOTHER LEAP IN SEM IMAGING TECHNOLOGY ETP 售害 ONCE AGAIN LEADS THE WAY}

- RS-232 Communication.

- Intuitive Joystick Operation.

- Optimum Bandwidth Selection.

- True TV Imaging without Edge Smearing.

- Retractable Design for Maximum Flexibility.

- Computer Interface via Windows Now Available.

- 8 Line - 40 Character LCD Display of Detector Status.

- Instant Save and Recall of Operating Conditions.

- Microprocessor Control of the Robinson Detector.

- Total Accessory Operation without using Valuable SEM Console Space.

- At Atomic Number 30, Discrimination is Better than 0.1 Atomic Numbers.

- $2000 \mathrm{~mm}$ Active Surface Area, $5 \mathrm{~mm}$ Thick at Beam Entry Point, 5:1 S/N at 1 PA, $10 \mathrm{KV}$.

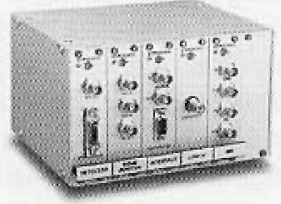

Extensive Accessory List including: Low Accelerating Voltage Operation,

SE-BSE Signal Mixing, Selected Phase Imaging, Motorized Retraction, Scanning

Transmission, Cathodoluminescence Detection and Much More...

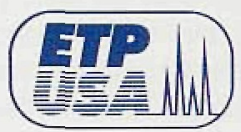

Electron Detectors, Inc. 1650 Holmes St. Livermore, CA 94550 - 1 (800) 8ETP USA • (510) 449-8534 - FAX (510) 449-8996 